\title{
Association of Inflammatory Markers with COVID-19 Outcome among Hospitalized Patients: Experience from a Tertiary Healthcare Center in Western India
}

Varatharajan SAKTHIVADIVEL a, Gopal Krishana BOHRA ${ }^{b}$, Nachimuthu MAITHILIKARPAGASELVIc , Satyendra KHICHAR, Mahadev MEENAd, Naveenraj PALANISAMY', Archana GAUR ${ }^{\mathrm{e}}$, Mahendra Kumar GARG ${ }^{\mathrm{b}}$

aDepartment of General Medicine, All India Institute of Medical Sciences, Bibinagar, Hyderabad, Telangana, India

bDepartment of General Medicine, All India Institute of Medical Sciences, Jodhpur, Rajasthan, India

'Department of Biochemistry, All India Institute of Medical Sciences, Jodhpur, Rajasthan, India

'Department of General Medicine, All India Institute of Medical Sciences, Bhopal, Madhya Pradesh, India

eDepartment of Physiology, All India Institute of Medical Sciences, Bibinagar, Hyderabad, Telangana, India

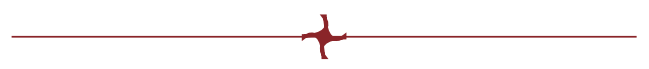

ABSTRACT-
Introduction: COVID-19 is a highly infectious disease and varies in the severity of presentation as well
as survival outcome due to varied inflammatory responses. Hence, the present study is aimed to evaluate the
role of inflammatory markers in predicting the outcome of COVID-19 in hospitalized patients.
Methods: A total of 272 confirmed COVID-19 patients were included in the study. Clinical and
demographic data were collected. Biochemical, hematological, and inflammatory markers were assessed in
all patients. Disease severity and primary outcome as survival and or mortality were recorded.
Results: Hematological indices and inflammatory markers were significantly higher among the
non-survivors. Interleukin-6 (IL-6) can differentiate non-survivors from survivors with $100 \%$ sensitivity
and $70.2 \%$ specificity, with a cut-off value of 79.6 in the receiver operator curve (ROC). As disease severity
was increasing, IL-6 and C-reactive protein (CRP) were significantly increased among patients. Survival

Address for correspondence:

Dr. Archana Gaur T, MD, Assistant Professor

Department of Physiology, All India Institute of Medical Sciences, Bibinagar, Hyderabad, Telangana, India, Pin 508126

Tel.: 91-9962053334, email: drarchana85@gmail.com

Article received on the $8^{\text {th }}$ of September 2021 and accepted for publication on the $16^{\text {th }}$ of December 2021 
analysis showed that an elevated level of IL-6 was significantly associated with mortality and Cox regression analysis showed the hazard ratio (HR) of IL-6 was 0.996 ( $P<0.007)$.

Conclusion: The results of the present study implicate that increased levels of IL-6 and CRP were significantly correlated with severity and mortality in COVID-19 patients. In addition, the dynamic measurement of neutrophil-to-lymphocyte (N/L) ratio, IL-6, and CRP in COVID-19 might be used as predictors of prognosis and outcome.

Keywords: inflammatory marker, COVID-19, outcome, mortality, ROC.

\section{INTRODUCTION}

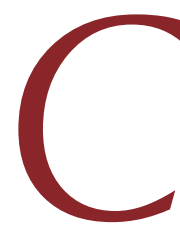

OVID-19 is caused by beta-Coronavirus, with the severe acute respiratory syndrome-Coronavirus-2 (SARS-CoV-2) being responsible for the current pandemic. COVID-19 infection can be either asymptomatic or present with severe and critical symptoms (1). The presenting symptoms may range from mild fever, runny nose, cough, and redness of eyes to chest pain, shortness of breath, and coma (2). The infection predominantly affects the respiratory system, but it can also have an impact on various other organs. This varied clinical presentation is attributed to a differences in the immune response of individuals (3). SARS-CoV-2 principally infects lower respiratory tract pneumocytes. Virus replication is associated with host cell pyroptosis, causing the release of damage-associated molecular patterns (DAMPs), which trigger the generation of proinflammatory cytokines and chemokines like type I interferons (IFN- $\alpha$ and IFN- $\beta$ ) that protect from any viral infection (4). The interferon released in young individuals may be responsible for inhibition of virus replication and hence, they usually have only a mild infection. However, in old individuals with multiple comorbidities, and due to immune system senescence, chronic low-grade systemic inflammation, termed inflammaging and delayed release of interferons, leads to further recruitment of neutrophils, macrophages, and monocytes (5). The defective immune response causes an overproduction of pro-inflammatory cytokines, known as cytokine storm, that damages the lung alveoli, causing severe acute respiratory syndrome (6), which in turn results in sepsis and multiple organ system damages.

Earlier studies observed higher levels of proinflammatory factors such as IL-2, IL-7, IL-10, IL-6, macrophage inflammatory protein $1 \alpha$ (MIP1 $\alpha)$, and tumour necrosis factor (TNF) in COVID-19 patients with intensive care support. (7). Lactate dehydrogenase (LDH), IL-6, CRP and creatinine were elevated in patients with respiratory failure (8). Elevated serum IL-6 levels were noted in patients with severe COVID-19 symptoms and can be an early predictor of patients' outcome (9).

The present study was done to assess the association of various inflammatory markers with the outcome of COVID-19 patients, so that therapeutic strategies against overactive cytokine response with immunomodulators, but in a balanced way with the adequate immune response to clear the pathogen, can be planned.

\section{MATERIAL AND METHODS}

The current research, a cross sectional observational study, was conducted between March 2020 and December 2020 at a tertiary care center in West India after obtaining the ethics approval of the Institute Ethics Committee (AIIMS/IEC/2020-21/2003). Two hundred seventy two patients aged $\geq 18$ years, who were admitted to specified wards/intensive care for COVID-19, have been enrolled in the study after obtaining their informed written consent. On their first visit to the hospital, a baseline assessment of variables was done, including socio-demographic profile and novel corona virus 2019 (nCoV-19) positive test confirmed by a RT-PCR test. Patients were categorized into asymptomatic, mild, moderate, and severe as per MoHFW (Ministry of Health and Family Welfare) criteria and asymptomatic, mild, moderate, severe, and critical as per $\mathrm{WHO}$ criteria (9). They were all managed as per the MoHFW guidelines (10).

Patients were observed for worsening signs of COVID-19 in the form of hypotension, sepsis, MODS, pneumonia, ARDS, respiratory failure, myocarditis, CAD, heart failure, acute kidney injury, DVT, pulmonary thrombo-embolism, and 
stroke. Blood samples were analyzed for complete hemogram, renal and liver function tests, CRP, IL-6, ferritin, D-dimer, LDH, and CK-Nac. Patients' outcome in terms of recovery and mortality was noted.

\section{Statistical analysis}

Data were analyzed using SPSS 22.0. Quantitative variables were represented as mean $\pm \mathrm{SD}$ or median. Qualitative data were presented as numbers and percentages. Chi-square test was used to find the difference among qualitative data. Man-Whitney $U$ test, a non-parametric test, was used to explore the difference between survivors

TABLE 1. Basic characteristics of COVID-19 patients

\begin{tabular}{|c|c|c|}
\hline Parameter & Frequency $(\mathrm{N}=272)$ & Percentage \\
\hline \multicolumn{3}{|l|}{ Gender } \\
\hline Male & 201 & 74 \\
\hline Female & 71 & 21 \\
\hline \multicolumn{3}{|l|}{ Comorbidities } \\
\hline Diabetes & 106 & 39 \\
\hline Hypertension & 207 & 76.1 \\
\hline IHD & 16 & 5.9 \\
\hline Asthma & 3 & 1.1 \\
\hline $\mathrm{CAD}$ & 4 & 1.5 \\
\hline CKD & 2 & 0.74 \\
\hline \multicolumn{3}{|l|}{ Outcome } \\
\hline Survivor & 28 & 10.3 \\
\hline Non-survivor & 244 & 89.7 \\
\hline \multicolumn{3}{|l|}{ Complications } \\
\hline Yes & 50 & 18.4 \\
\hline No & 222 & 81.6 \\
\hline \multicolumn{3}{|l|}{ Severity } \\
\hline \multicolumn{3}{|l|}{ MoHFW } \\
\hline Asymptomatic & 44 & 16.2 \\
\hline Mild & 109 & 40.1 \\
\hline Moderate & 30 & 11.0 \\
\hline Severe & 89 & 32.7 \\
\hline \multicolumn{3}{|l|}{ WHO } \\
\hline Asymptomatic & 44 & 16.2 \\
\hline Mild & 109 & 40.1 \\
\hline Moderate & 30 & 11.0 \\
\hline Severe & 48 & 17.6 \\
\hline Critical & 41 & 15.1 \\
\hline
\end{tabular}

and non-survivors. Overall survival was assessed by Cox proportional hazards regression models. Receiver-operating characteristic (ROC) was done to find the cut-off value of $\mathrm{N} / \mathrm{L}$ ratio, IL- 6 , and CRP to predict the outcome in COVID-19 patients. The P-value of less than 0.05 was considered statistically significant.

\section{RESULTS}

Table 1 shows the basic characteristic of COVID-19 patients (73.9\% males and $26 \%$ females). Hypertension was the commonest comorbidity, followed by diabetes and ischemic

TABLE 2. Predictors of mortality among COVID-19 patients

\begin{tabular}{|c|c|c|c|}
\hline Parameter & $\begin{array}{l}\text { Survivors } \\
(\mathrm{N}=\mathbf{2 4 4})\end{array}$ & $\begin{array}{l}\text { Non-survivors } \\
(\mathrm{N}=\mathbf{2 8})\end{array}$ & $\begin{array}{l}P \\
\text { value }\end{array}$ \\
\hline \multicolumn{4}{|l|}{ Gender } \\
\hline Male & $180(73.8)$ & $21(75)$ & 0.546 \\
\hline Female & $64(26.2)$ & $7(25)$ & \\
\hline \multicolumn{4}{|c|}{ Comorbidities } \\
\hline \multicolumn{4}{|c|}{ Diabetes mellitus } \\
\hline Yes & $93(38.1)$ & $13(46.4)$ & 0.256 \\
\hline No & $151(61.9)$ & $15(53.6)$ & \\
\hline \multicolumn{4}{|l|}{ Hypertension } \\
\hline Yes & $179(73.4)$ & $28(100)$ & 0.001 \\
\hline No & $65(26.6)$ & $0(0)$ & \\
\hline \multicolumn{4}{|c|}{ Complications } \\
\hline Yes & $23(9.4)$ & $28(100)$ & 0.000 \\
\hline No & $221(90.6)$ & 0 & \\
\hline \multicolumn{4}{|l|}{ Smoking } \\
\hline Yes & $4(1.6)$ & $0(0)$ & 0.646 \\
\hline No & $240(98.4)$ & $28(100)$ & \\
\hline \multicolumn{4}{|l|}{ Symptoms } \\
\hline Fever & $123(50.4)$ & $19(67.9)$ & 0.059 \\
\hline Cough & $116(57.5)$ & $15(53.6)$ & 0.342 \\
\hline Respiratory & $65(26.6)$ & $19(67.9)$ & 0.000 \\
\hline \multicolumn{4}{|l|}{ distress } \\
\hline Sore throat & $9(3.7)$ & $0(0)$ & 0.370 \\
\hline Headache & $8(3.3)$ & $0(0)$ & 0.414 \\
\hline Muscle pain & $7(2.9)$ & $1(3.6)$ & 0.586 \\
\hline Others & $6(2.5)$ & $0(0)$ & 0.518 \\
\hline Diarrhoea & $3(1.2)$ & $0(0)$ & 0.721 \\
\hline Cold & $0(0)$ & $0(0)$ & - \\
\hline
\end{tabular}




\begin{tabular}{lccc}
\hline Parameter & Survivors $(\mathbf{N}=\mathbf{2 4 4})$ & Non-survivors $(\mathbf{N}=\mathbf{2 8})$ & $\boldsymbol{P}$ value \\
\hline Age (years) & $55.67 \pm 15.46$ & $64.11 \pm 13.87$ & 0.005 \\
Total leucocyte count $(/ \mu \mathrm{L})$ & $7329.88 \pm 3707.56$ & $9372.86 \pm 3924.63$ & 0.007 \\
Neutrophil count $(/ \mu \mathrm{L})$ & $5039.22 \pm 3718.99$ & $7747.74 \pm 3409.68$ & 0.000 \\
Lymphocyte count $(/ \mu \mathrm{L})$ & $1670.83 \pm 866.12$ & $1098.64 \pm 1157.08$ & 0.000 \\
Neutrophil lymphocyte ratio & $4.34 \pm 5.35$ & $13.33 \pm 10.09$ & 0.000 \\
Platelet count $\left(\times 10^{3}\right)$ & $244.38 \pm 94.1$ & $199.0 \pm 64.1$ & 0.041 \\
CRP $(\mathrm{mg} / \mathrm{dL})$ & $46.1 \pm 54.35$ & $93.96 \pm 66.31$ & 0.000 \\
Ferritin $(\mathrm{ng} / \mathrm{mL})$ & $376.49 \pm 455.68$ & $807.68 \pm 583.98$ & 0.000 \\
AST $(\mathrm{U} / \mathrm{L})$ & $39.87 \pm 32.22$ & $48.99 \pm 21.45$ & 0.002 \\
ALT $(\mathrm{U} / \mathrm{L})$ & $35.77 \pm 29.79$ & $43.83 \pm 28.07$ & 0.014 \\
Urea $(\mathrm{mg} / \mathrm{dL})$ & $32.47 \pm 23.71$ & $53.71 \pm 43$ & 0.000 \\
Creatinine $(\mathrm{mg} / \mathrm{dL})$ & $1.13 \pm 0.84$ & $3.25 \pm 9.99$ & 0.001 \\
IL-6 $(\mathrm{pg} / \mathrm{mL})$ & $106.25 \pm 176.83$ & $217.78 \pm 45.23$ & 0.000 \\
D-dimer $(\mu \mathrm{g} / \mathrm{mL})$ & $1.84 \pm 6.36$ & $9.47 \pm 27.53$ & 0.000 \\
LDH $(\mathrm{U} / \mathrm{L})$ & $290.27 \pm 137.23$ & $390.16 \pm 238.36$ & 0.001 \\
CKNAC $(\mathrm{U} / \mathrm{L})$ & $82.55 \pm 76.99$ & $167.88 \pm 225.66$ & 0.031 \\
\hline
\end{tabular}

TA B L E 3. Inflammatory profile and biochemical parameters among survivors and non-survivors

\begin{tabular}{|c|c|c|c|c|c|c|}
\hline Parameter & AUC (95\% CI) & Sensitivity & Specificity & Cut-off value & P value & \\
\hline IL-6 & $0.864(0.822-0.906)$ & 100 & 70.2 & 79.6 & 0.000 & \\
\hline CRP & $0.734(0.642-0.827)$ & 68.0 & 64.8 & 48.8 & 0.000 & \\
\hline Ferritin & $0.790(0.709-0.871)$ & 75.0 & 71.3 & 444.7 & 0.000 & \\
\hline $\mathrm{TC}$ & $0.654(0.545-0.674)$ & 60.7 & 64.3 & 7175 & 0.007 & \\
\hline $\mathrm{N} / \mathrm{L}$ ratio & $0.842(0.761-0.924)$ & 89.3 & 74.2 & 4.4 & 0.000 & \\
\hline D-dimer & $0.737(0.627-0.847)$ & 71.4 & 65.6 & 0.78 & 0.000 & $\begin{array}{l}\text { TABLE 4. Performance } \\
\text { criteria of inflammatory }\end{array}$ \\
\hline LDH & $0.695(0.597-0.793)$ & 67.9 & 66.8 & 312 & 0.001 & $\begin{array}{l}\text { markers in discriminating } \\
\text { the outcome of COVID-19 }\end{array}$ \\
\hline
\end{tabular}

heart disease (IHD). Of the total number of patients, $89.7 \%$ survived and only $18.4 \%$ had complications. According to WHO criteria of severity, $17.3 \%$ were severely ill and $13.2 \%$ critically ill, whereas MoHFW criteria included both in a single category (severe disease). The presence of hypertension and complications were significantly higher in the non-survival group. The presence of respiratory distress among non-survivors was also associated with poor outcome. (Table 2).

Hematological indices, including total leucocyte count, neutrophil count, and $\mathrm{N} / \mathrm{L}$ ratio $(\mathrm{P}<0.0001)$, were significantly elevated among non-survivors. Inflammatory markers, including
IL-6 $(\mathrm{P}<0.0001), \mathrm{CRP}(\mathrm{P}<0.0001)$ and ferritin, were also higher among non-survivors. Levels of aspartate transaminase (AST), alanine transaminase (ALT), urea, creatinine, D-dimer, LDH, and $\mathrm{N}$-acetylcystein creatinkinase (CK-Nac) were also significantly increased among patients with a poor outcome (Table 3). The receiver operator curve analysis of IL-6, CRP, and N/L ratio showed a good sensitivity of $100 \%, 80 \%$ and $68 \%$, respectively with a cut-off of $79.9,48.8$, and 4.4 , respectively in predicting the outcome of COVID-19. Of all markers, IL-6 appears to have $100 \%$ sensitivity and $70.2 \%$ specificity (Figures 1 and 2, Table 4).

Patients with COVID-19 were divided into two groups, according to IL-6 ( $\leq 79$ and $>79)$ and 
FIGURE 1. ROC curve of laboratory markers as predictors of COVID-19 outcome

\section{FIGURE 2.} ROC curve of laboratory markers as predictors of COVID-19 outcome
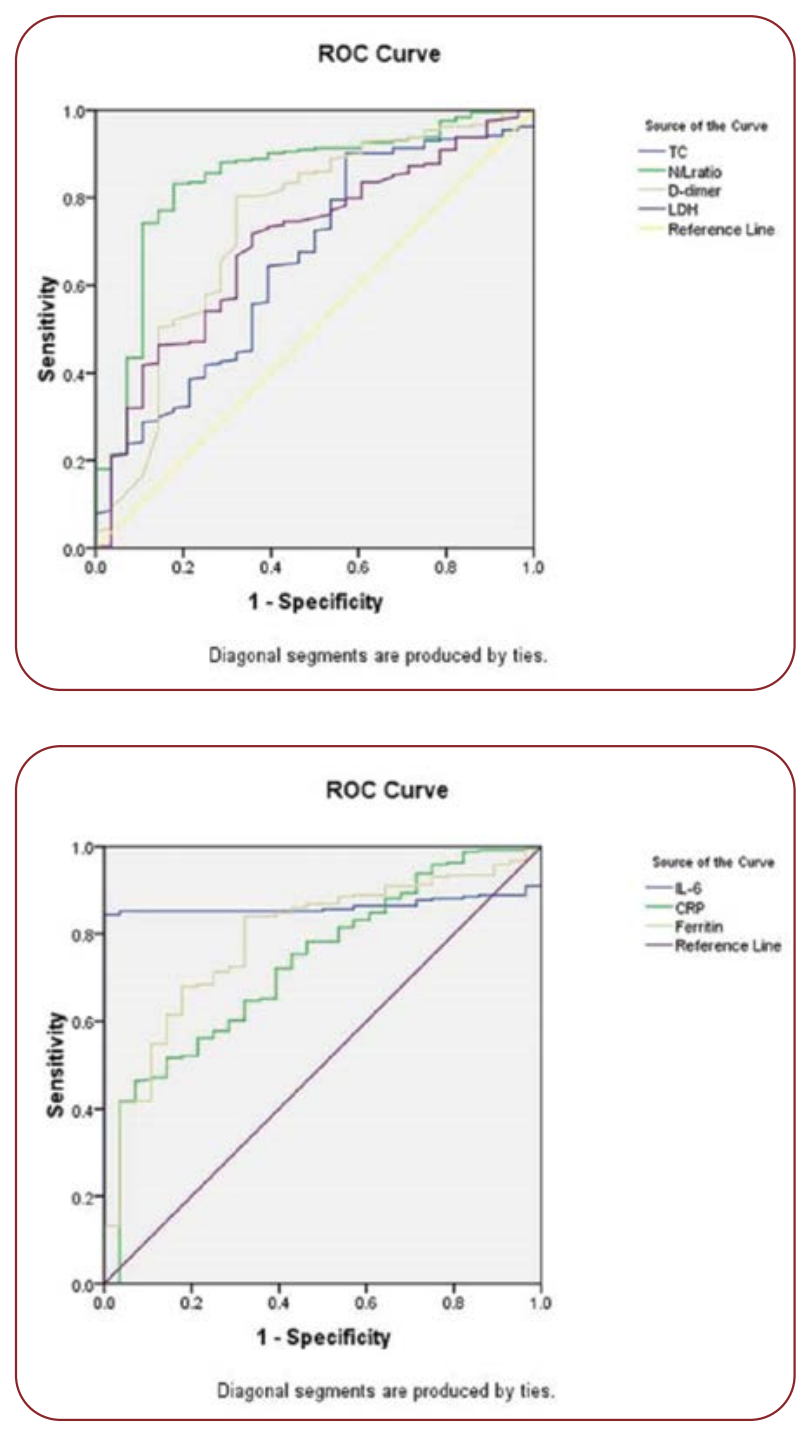

CRP ( $\leq 48.8$ and $>48.8$ ) levels. Survival analysis showed that elevated levels of IL- 6 and CRP were significantly associated with mortality. The unadjusted HRs of IL- 6 and CRP for mortality were $0.996(\mathrm{Cl} 0.994-0.999, \mathrm{P}<0.007)$ and 1.1 (Cl 1.002-1.012, $\mathrm{P}<0.008)$, and independent predictor of mortality (Figure 3 ).

\section{DISCUSSION}

In our study, the male gender was mostly affected by COVID-19. Patients' mean age was also significantly higher among non-survivors when compared to survivors. Hypertension (76\%) was the most common comorbidity found in COVID-19 infected patients, followed by diabetes mellitus, IHD, asthma, cardiovascular disease (CVD), and chronic kidney disease (CKD). Ac- cording to our findings, hypertension was highly associated with complications, similarly to those obtained by Yang J. et al (12). The most prevalent symptoms included fever, cough, and respiratory distress, followed by sore throat, muscle pain, headache and diarrhea. When compared to survivors, the incidence of respiratory distress was substantially higher among non-survivors. The current study showed that the mean age of non-survivors was higher than survivors. Previous studies had demonstrated that older age, male gender, hypertension, diabetes mellitus, and cardiovascular disease represented major risk factors for severe COVID-19 and mortality $(13,14)$.

The values of leukocyte count, neutrophil count, and $\mathrm{N} / \mathrm{L}$ ratio were significantly increased and platelet count was significantly decreased in non-survivors, indicating the inflammatory state of SARS-CoV-2 infection. A study demonstrated that elevated $\mathrm{N} / \mathrm{L}$ ratio and decreased platelet count were useful predictors for severity and mortality in COVID-19 patients admitted in intensive care units (15). In our study, N/L ratio had good sensitivity and specificity in discriminating clinical outcomes in COVID-19 patients. Yang et al found that $\mathrm{N} / \mathrm{L}$ ratio was an independent predictor of severity and poor outcome in COVID-19 patients (16).

Inflammatory responses play a vital role in the course of COVID-19 and many inflammatory markers have been identified for measuring the severity and may be a potential treatment target (17).

Interleukin-6 is an acute-phase inflammatory cytokine that becomes elevated within two to three hours of infection and reflects the severity, degree of lung inflammation and association with poor clinical outcome in COVID-19 patients $(18,19)$. We found a significant elevation of IL-6 in non-survivors when compared to survivors. Furthermore, it has been reported that an IL-6 level $>240 \mathrm{pg} / \mathrm{mL}$ was associated with poor outcome in COVID-19 patients (20). Also, IL-6 has the greatest sensitivity in predicting mortality among non-survivors in COVID-19 patients. In our study, as severity increased, the mean value of IL-6 was significantly increased from asymptomatic to severe cases. Similarly, another study has shown that IL-6 was significantly increased in the group with critically severe disease when compared to that with severe disease (21). 
A

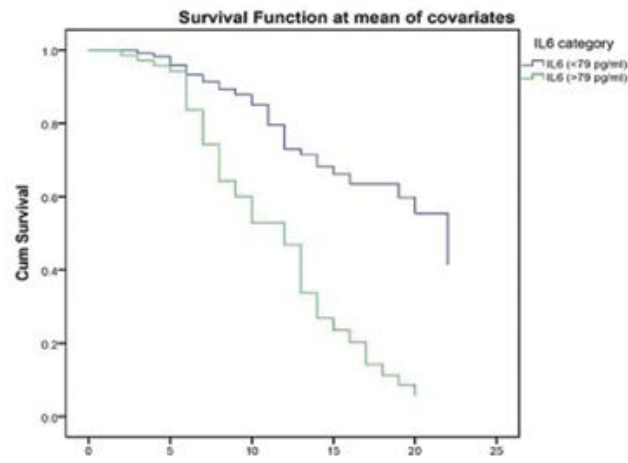

B

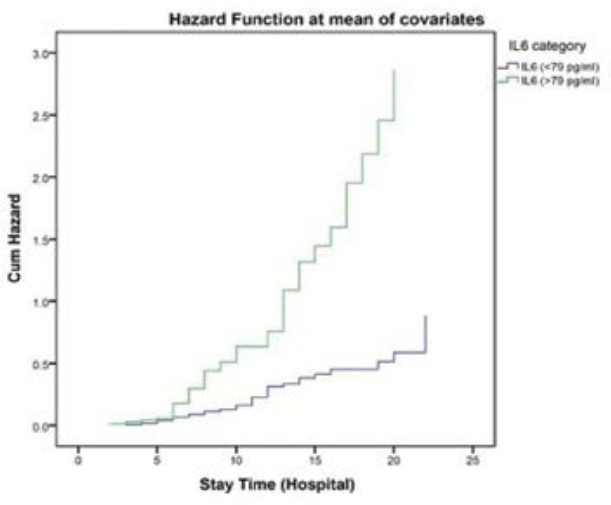

C

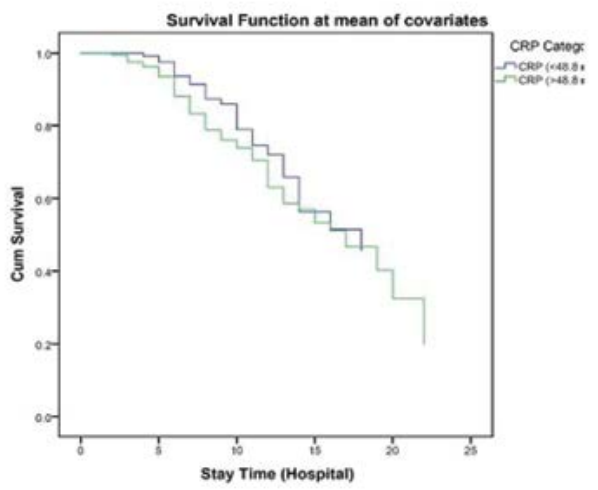

D

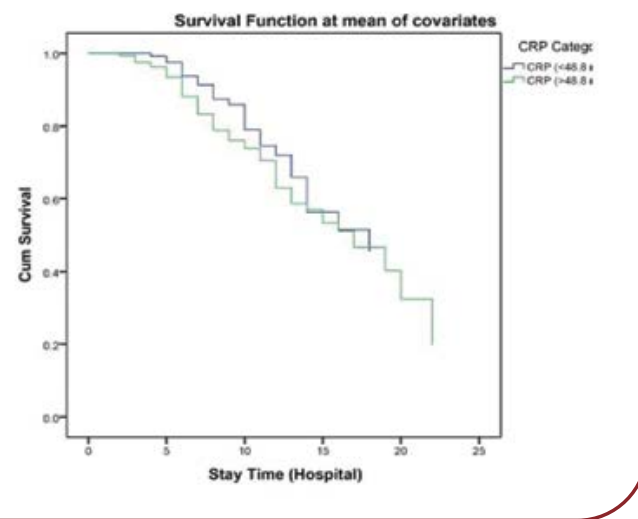

FIGURE 3. Cox proportional hazard ratio showing the relation between mortality and COVID-19 based on levels of interleukin-6 (IL-6) and C-reactive protein (CRP)
In our study, IL-6 was the best marker for assessing the severity shown by ROC analysis, with an AUC of 0.835. Survival analysis showed that elevated levels of IL-6 were significantly associated with mortality and Cox regression analysis showed the HR of IL-6 was 0.996, which predicted mortality among COVID-19 patients. Furthermore, COVID-19 patients with IL-6 levels greater than $32.1 \mathrm{pg} / \mathrm{mL}$ were more likely to experience severe complications, and IL- 6 was an independent forecaster of COVID-19 severity (22). Liu et al found that the mortality rate was higher in patients with an IL-6 level $>7 \mathrm{pg} / \mathrm{mL}$. Cox proportional hazards and logistic regression analyses showed that mortality rate and disease severity were greater in patients with elevated levels of IL-6 (23). Hence, it could be used as a potential biomarker for the assessment of severity and mortality in COVID-19 patients.

C-reactive protein is an acute-phase protein produced along with IL-6, an indicator of inflammation and tissue damage; CRP levels were also found to be elevated among non-survivors. C-reactive protein is significantly correlated with ferritin and IL- 6 and suggestive of elevated inflammatory response in COVID-19. In our study, as severity increased, the mean value of CRP was significantly increased from asymptomatic to severe cases. Hence, we determined a cut-off value for CRP which may help in predicting mortality in COVID-19 patients. The CRP value of $>48 \mathrm{mg} / \mathrm{dL}$ increases the risk of mortality among the patients. In line with our study, Liu et al have also stated that CRP $>41.8 \mathrm{mg} / \mathrm{dL}$ was associated with disease severity (23). In the present study, cumulative hazard analyses showed that elevated CRP levels were significant predictors of mortality in COVID-19 patients. Elevation of CRP is a predictive biomarker for severity and mortality among COVID-19 patients and those who require ICU admission. Thus, our results confirmed those provided by several earlier studies (24-26).

Not only these cytokines are heightened in severe symptoms but they are also promising biomarkers to differentiate between mild, moderate, and severe cases. Exacerbated inflammatory responses in SARS-CoV-2 are associated with the risk of developing severe symptoms such as lung injury, ARDS, multi-organ failure, and poor prognosis (27). In our study, biochemical parameters, including AST, ALT, urea, creatinine, LDH, CK-Nac, D-dimer, and ferritin, were significantly increased 
among non-survivors when compared to survivors. These abnormalities suggest that SARS-CoV-2 infection is associated with hepatic, renal, and myocardial injury as well as other organ damages (28). Besides, Wang et al (29) showed that AST, ALT, LDH, and D-dimer levels were significantly increased in critical COVID-19 patients. Ferritin is the mediator of inflammatory response released along with CRP and IL-6 and it is associated with fatal outcomes in COVID-19 patients. Epidemiological studies demonstrated that COVID-19 patients had a rapid activation of the innate immune response through the elevation of acute phase reactants such as ESR, CRP, IL-6, and ferritin (30).

The fact that our research was conducted in single tertiary healthcare centre, on a relatively small sample size of only 272 patients, was a limitation in our study.

\section{CONCLUSION}

nterleukin- 6 has been contemplated as the most accurate predictor of severity and morta- lity in COVID-19 patients among routine investigations. Furthermore, measuring IL-6 levels in the blood can aid in the development of therapeutics targets for IL-6 or its signaling for the treatment of COVID-19 patients. The present results implicated that increased IL- 6 and CRP levels were significantly correlated with severity and mortality in COVID-19 patients. In addition, the dynamic measurements of N/L ratio, IL-6, and CRP in COVID-19 patients might be used as predictors of prognosis, outcome, and therapeutic response. These inflammatory markers and clinical characteristics need further investigations to a large scale.

\section{Conflicts of interest: none declared.}

Financial support: none declared.

Acknowledgments: We would like to thank the Institute for providing support and facilities for our study as well as the faculties, students and staffs involved in COVID-19 care management.

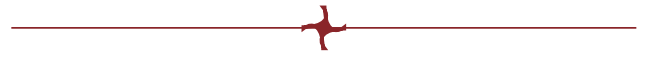

\section{$\mathbf{R}_{\text {eferences }}$}

1. Gao Z, Xu Y, Sun C, et al. A systematic review of asymptomatic infections with COVID-19.

J Microbiol Immunol Infect 2021;54:12-16.

2. Shi $\mathbf{H}$, Han $X$, Jiang $\mathbf{N}$, et al. Radiological findings from 81 patients with COVID-19 pneumonia in Wuhan, China: a descriptive study.

Lancet Infect Dis 2020;20:425-434.

3. Fajgenbaum DC, June $\mathrm{CH}$. Cytokine Storm. N Engl J Med 2020;383:2255-2273.

4. Mishra KP, Singh AK, Singh SB. Hyperinflammation and Immune Response Generation in COVID-19. Neuroimmunomodulation 2020;27:80-86.

5. Felsenstein S, Herbert JA, McNamara PS, Hedrich CM. COVID-19: Immunology and treatment options. Clin Immunol Orlando Fla 2020;215:108448.

6. Hazeldine J, Lord JM.

Immunesenescence: A Predisposing Risk Factor for the Development of COVID-19? Front Immunol (Internet). 2020 (cited 2021 Jul 2);11. Available from: https://www.frontiersin.org/ articles/10.3389/fimmu.2020.573662/full.

7. Arnett S. What we know about COVID-19 and the inflammatory response. 2021 Mar (cited 2021 Jun 27); Available from:

https://www.drugtargetreview.com/ article/85765/what-we-know-aboutcovid-19-and-the-inflammatory-response/

8. Herold T, Jurinovic V, Arnreich C, et al. Elevated levels of IL- 6 and CRP predict the need for mechanical ventilation in COVID-19. J Allergy Clin Immunol 2020;146:128-136.e4.

9. Sun $H$, Guo $P$, Zhang $L$, Wang $F$. Serum Interleukin-6 Concentrations and the Severity of COVID-19 Pneumonia: A Retrospective Study at a Single Center in Bengbu City, Anhui Province, China, in January and February 2020. Med Sci Monit Int Med J Exp Clin Res 2020;26:e926941-1-e926941-6.

10. https://www.who.int/publications/i/item/ WHO-2019-nCoV-clinical-2021-1

11. CLINICAL MANAGEMENT PROTOCOL: COVID-19 (Internet). Available from: https://www.mohfw.gov.in/pdf/ ClinicalManagementProtocolforCOVID19dated2706202.pdf

12. Yang J, Zheng $Y$, Gou $X$, et al. Prevalence of comorbidities and its effects in patients infected with SARS-CoV-2: a systematic review and meta-analysis. Int J Infect Dis 2020;94:91-95.

13. Qin C, Zhou L, Hu Z, et al. Dysregulation of Immune Response in Patients With Coronavirus 2019 (COVID-19) in Wuhan, China. Clin Infect Dis 2020;71:762-768.

14. Li X, Xu S, Yu M, et al. Risk factors for severity and mortality in adult COVID-19 inpatients in Wuhan. J Allergy Clin Immunol 2020;146:110.

15. Liao D, Zhou F, Luo L, et al. Haematological characteristics and risk factors in the classification and prognosis evaluation of COVID-19: a retrospective cohort study. Lancet Haematol 2020;7:e671-e678.

16. Yang A-P, Liu J, Tao W, Li H. The diagnostic and predictive role of NLR, d-NLR and PLR in COVID-19 patients. Int Immunopharmacol 2020;84:106504.

17. Mehta P, McAuley DF, Brown M, et al. COVID-19: consider cytokine storm syndromes and immunosuppression. The Lancet 2020;395:1033-1034.

18. Bhandari S, Rankawat G, Singh A, et al. Evaluation of interleukin- 6 and its association with the severity of disease in COVID-19 patients. 
Indian J Med Spec 2020;11:132.

19. Santa Cruz A, Mendes-Frias A, Oliveira AI, et al. Interleukin-6 Is a Biomarker for the Development of Fatal Severe Acute Respiratory Syndrome Coronavirus 2 Pneumonia. Front Immunol (Internet). 2021 (cited 2021 Jul 3);12. Available from: https://www.frontiersin.org/ articles/10.3389/fimmu.2021.613422/full.

20. Broman N, Rantasärkkä K, Feuth T, et al. IL- 6 and other biomarkers as predictors of severity in COVID-19. Ann Med 2021;53:410-432.

21. Wang M, Fan Y, Chai Y, et al. Association of Clinical and Immunological Characteristics With Disease Severity and Outcomes in 211 Patients With COVID-19 in Wuhan, China.

Front Cell Infect Microbiol (Internet). 2021 (cited 2021 Jul 3);11. Available from: https://www.frontiersin.org/ articles/10.3389/fcimb.2021.667487/full.

22. Liu Z, Li J, Chen D, et al. Dynamic
Interleukin-6 Level Changes as a Prognostic Indicator in Patients With COVID-19.

Front Pharmacol 2020;11:1093.

23. Liu F, Li L, Xu M, et al. Prognostic value of interleukin-6, C-reactive protein, and procalcitonin in patients with COVID-19. J Clin Virol Off Publ Pan Am Soc Clin Virol 2020;127:104370.

24. Canovi S, Besutti G, Bonelli E, et al. The association between clinical laboratory data and chest CT findings explains disease severity in a large Italian cohort of COVID-19 patients. BMC Infect Dis 2021;21:157.

25. Kazemi E, Soldoozi Nejat R, et al. The laboratory findings and different COVID-19 severities: a systematic review and meta-analysis. Ann Clin Microbiol Antimicrob 2021;20:17.

26. Nadeem R, Elhoufi AM, Iqbal NE, et al. Prediction of Cytokine Storm and Mortality in Patients with COVID-19 Admitted to ICU: Do Markers
Tell the Story?

Dubai Med J 2021;4:142-150.

27. Mokhtari T, Hassani F, Ghaffari N, et al. COVID-19 and multiorgan failure: A narrative review on potential mechanisms. J Mol Histol 2020;51:613-628.

28. Bairwa M, Kumar R, Beniwal K, et al. Hematological profile and biochemical markers of COVID-19 non-survivors: A retrospective analysis.

Clin Epidemiol Glob Health 2021;11:100770.

29. Wang D, Li R, Wang J, et al. Correlation analysis between disease severity and clinical and biochemical characteristics of 143 cases of COVID-19 in Wuhan, China: a descriptive study. BMC Infect Dis 2020;20:519.

30. Huang I, Pranata R, Lim MA, et al. C-reactive protein, procalcitonin, D-dimer, and ferritin in severe coronavirus disease-2019: a meta-analysis. Ther Adv Respir Dis 2020;14:1753466620937175. 